\title{
CORRIGENDUM
}

\section{Validity and reliability of an online self-report 24-hour dietary recall method (Intake24): A doubly-labelled water study and repeated measures analysis - CORRIGENDUM}

Emma Foster ${ }^{1}$, Clement Lee ${ }^{2,3}$, Fumiaki Imamura ${ }^{4}$, Stefanie E. Hollidge ${ }^{4}$, Kate L. Westgate ${ }^{4}$, Michelle C. Venables ${ }^{5}$, Ivan Poliakov ${ }^{6}$, Maisie K. Rowland ${ }^{1}$, Timur Osadchiy ${ }^{6}$, Jennifer C. Bradley ${ }^{1}$, Emma L. Simpson ${ }^{6}$, Ashley J. Adamson ${ }^{1}$, Patrick Olivier ${ }^{7}$, Nick Wareham ${ }^{4}$, Nita G. Forouhi ${ }^{4}$ and Soren Brage 4

${ }^{1}$ Human Nutrition Research Centre, Institute of Health and Society, Newcastle University, Newcastle upon Tyne, UK

${ }^{2}$ School of Mathematics, Statistics and Physics, Newcastle University, Newcastle upon Tyne, UK

${ }^{3}$ Department of Mathematics and Statistics, Lancaster University, Lancaster, UK

${ }^{4}$ MRC Epidemiology Unit, University of Cambridge, Cambridge, UK

${ }^{5} \mathrm{MRC}$ Elsie Widdowson Laboratory, Cambridge, UK

${ }^{6}$ Open Lab, School of Computing Science, Newcastle University, Newcastle upon Tyne, UK

${ }^{7}$ Faculty of Information Technology, Monash University, Clayton, VTC, Australia

Journal of Nutritional Science (2019), vol. 8, e41, page 1 of 1

doi:10.1017/jns.2019.38

doi:10.1017/jns.2019.20, Published online by Cambridge University Press, 30 August 2019

Key words: Dietary assessment: Online 24-h dietary recall: Doubly labelled water: Validation: Repeatability: Reliability: UK adults:

Corrigendum

In the aforementioned article, the affiliation for Soren Brage is incorrect. The correct affiliation for Soren Brage is: MRC Epidemiology Unit, University of Cambridge, Cambridge, UK

The authors would like to apologise for this error.

\section{Reference}

Foster E., Lee C., Imamura F., et al. (2019) Validity and reliability of an online self-report 24-h dietary recall method (Intake24): a doubly labelled water study and repeated-measures analysis. J Nutr Sci 8, E29. doi:10.1017/jns.2019.20

\footnotetext{
*Corresponding author: Emma Simpson, email Emma.Simpson@ncl.ac.uk
} 